\title{
DAUPHINÉ TWIN OBSERVATION IN QUARTZ USING PIEZO OR ELECTRO-OPTIC EFFECTS
}

\author{
G. DOLINO \\ Laboratoire de Spectrométrie Physique (*), BP 53, 38041 Grenoble Cedex, France \\ (Reçu le 26 mai 1975, accepté le 24 juin 1975)
}

\begin{abstract}
Résumé. - L'influence des macles du quartz sur les tenseurs piézo-optique et électro-optique est examinée, du point de vue des symétries. Etant donné que certains coefficients changent de signe avec les macles, on peut utiliser ces effets pour observer les macles. Plusieurs possibilités expérimentales utilisant l'application d'une contrainte uniaxiale ou d'un champ électrique sont discutées et des résultats expérimentaux sont présentés pour des macles du Dauphiné, qui ne sont pas observables par les méthodes optiques ordinaires.
\end{abstract}

Abstract. - The influence of twinning in quartz on the piezooptic and the electrooptic tensors is examined from the point of view of symmetry. Since some tensor coefficients change their sign with twinning one can make observations of twins using these effects. Several experimental dispositions using uniaxial stress or an electric field are discussed and experimental results are presented for Dauphiné twins, which are not visible by ordinary optical methods.

The stable phase of silicon dioxide $\mathrm{SiO}_{2}$ under ordinary conditions is quartz (low quartz or $\alpha$-quartz to distinguish it from another closely related hightemperature phase called high quartz or $\beta$ quartz) [1]. Since the beginning of the XIXth century it has been known that quartz possesses several kinds of penetration twins (with parallel axis) [2,3]. The species which changes the sign of optical rotatory power is called Brazil or optical twin. Another kind called electrical or Dauphiné twin is associated with a change of the piezoelectric constant and so is quite interesting for industrial use. In natural crystals, twinning is sometimes shown by an anomalous disposition of faces, but even well-shaped crystals can be twinned. Brazil twins are easily detected by optical means, using the change of rotatory power. The usual way to detect Dauphiné twins is by etching with hydrofluoric or similar acid. They have been also observed by X-ray topography [4] or by electron microscopy [5]. Recently, we have reported an optical method to detect Dauphiné twins using second harmonic generation of light [6]. In this paper we discuss the use of other non-linear optical methods using electro-optic and piezo-optic effects (observation of Dauphiné

(*) Associé au C. N. R. S. twinning using piezo-optic effects seems to have been made previously only by Aizu and Irai [7]). After recalling some crystallographic aspects of twinning and their relation to physical properties of crystals, we shall examine several experimental disposition to observe Dauphiné twinning, using uniaxial stress or electric field, and then present some results that we have obtained on quartz samples.

1. Influence of twinning on optical properties. The different twinning possibilities can be deduced from the knowledge of crystal symmetry. $\alpha$-quartz belongs to the symmetry class 32 (one rotation axis $\mathrm{C}_{3}$ of order 3.3 perpendicular axes $\mathrm{C}_{2}$ of order 2 , $O Z$ is along $\mathrm{C}_{3}$ and $O X$ along one of $\mathrm{C}_{2}$ axes). $\beta$-quartz belongs to 622 and $O Z$ becomes a rotation axis of order 6 , there are also 3 new perpendicular axes $C_{2}^{\prime}$ of order 2. But the lattice alone is more symmetrical and belongs to $6 / \mathrm{mm}$. The supplementary symmetry elements include a center of inversion $i$ and consequently several symmetry planes $\left(\sigma_{\mathrm{h}}\right.$ perpendicular to $O Z, \sigma_{\mathrm{v}}$ and $\sigma_{\mathrm{v}}$, perpendicular to $O X$ and $O Y$ respectively). When we go from the lattice symmetry to $\beta$-quartz the inversion $\mathrm{i}$, and the symmetry planes $\sigma$ give way to two possible enantiomorphic forms, where the atomic helicoidal arrangements are related by the lost symmetry operations. Following G. Frie- 
del [8], this is a case of holoaxial hemiedry. In going from $\beta$ to $\alpha$ phase, the rotation axis of order 6 becomes a rotation axis of order 3 , so losing a rotation of order 2 around $O Z$.

There are two possible orientations for $O X$, giving way to the possibility of Dauphiné twinning (Tetartoedry). In $\alpha$ phase Brazil twins are related by a plane of symmetry which is $Y O Z$. Not only is the rotatory power changed, but the polarity of $O X$ axis is also changed. When $O X$ keeps the same orientation, and only the rotatory power is changed, one has the combined law of twinning. Macroscopic physical properties are described by tensors, invariant under the symmetry operation of the crystal. Furthermore, if we introduce the symmetry operations of the $\beta$ phase or of the lattice (associated to the different twinnings), some tensor coefficients are invariant, while others are changed. Some physical properties are therefore changed by twinning. For example, the optical rotatory power associated with an axial second order tensor changes sign when the hand of the axis is changed, as in Brazil twins. A symmetric second rank tensor, such as the one giving refractive indices and birefringence is not changed by either kind of twinning. But if we go to higher rank tensors, such as those describing nonlinear optical effects (second harmonic generation [9], electro or piezo-optic effects [2]) some changes are produced by twinning, and in particular by Dauphiné twinning. Twinning is often the consequence of a phase transition of 2nd order or nearly 2 nd order, in which one can define an order parameter of tensorial nature. There is, in general, a decrease of symmetry in going from a high temperature to a low temperature phase, leaving several possibilities for the orientation of the order parameter producing twins or domains. Depending upon the symmetry of the high temperature phase and on the symmetry of the order parameter, physical properties can be changed or not.

A systematic search for these effects has been made by Aizu [10]. Recently, we have made a measurement of the order parameter of quartz by second harmonic generation and we have discussed its relation to other physical properties [11]. The order parameter $\eta$ belongs to the $B_{1}$ representation and changes its sign by a $180^{\circ}$ rotation around $O Z$. Physical properties transforming as $B_{1}$ can be expressed as an odd function of $\eta$, the other as an even function of $\eta$.

We come back to the problem of observing optically Dauphiné twins in quartz. Optical properties (neglecting rotatory power which is of importance only for light propagating along $O Z$ ) are described by the ellipsoid of refractive indices $n$. The equation of this is [2]

$$
\begin{aligned}
B_{11} x^{2}+B_{22} y^{2}+B_{33} z^{2}+2 B_{23} y z+ \\
+2 B_{13} x z+2 B_{12} x y=1
\end{aligned}
$$

where $x, y, z$ are the coordinate of a vector of modulus $n$, parallel to the electric induction $D$ of the light beam.
The linear electro-optic tensor $r_{i j k}$ (Pockels effect) describing the change of optical properties under the application of an electric field $E_{k}$ is defined by (with $i$, $j, k=x$ to $z$ or 1 to 3$)$ :

$$
B_{i j}^{\prime}=B_{i j}+r_{i j k} E_{k}
$$

$r_{i j k}$ is a 3 rd rank tensor, different from zero only in non centrosymmetric crystals. In quartz, it reduces to :

$$
\begin{array}{cccccc}
r_{111} & -r_{111} & 0 & r_{123} & 0 & 0 \\
0 & 0 & 0 & 0 & -r_{123} & -2 r_{111} \\
0 & 0 & 0 & 0 & 0 & 0
\end{array}
$$

When there is Dauphiné twinning $x$ and $y$ are changed to $-x$ and $-y$. So only $r_{111}$ changes its sign, while $r_{123}$ is not affected. The same is true for the component $e_{111}$ of the piezoelectric tensor and for $d_{111}$ of the second harmonic generation tensor.

In the same way change of optical properties under the application of a stress $\sigma_{k l}$ is defined by the piezooptice tensor $q_{i j k l}$ defined by :

$$
B_{i j}^{\prime}=B_{i j}+q_{i j k l} \sigma_{k l}
$$

In quartz this tensor takes the following form (using Voigt convention $x x=1, y y=2, z z=3$, $y z=4, x z=5, x y=6$ for subscript)

$\begin{array}{cccccc}q_{11} & q_{12} & q_{13} & q_{14} & 0 & 0 \\ q_{12} & q_{11} & q_{13} & -q_{14} & 0 & 0 \\ q_{31} & q_{31} & q_{33} & 0 & 0 & 0 \\ q_{41} & -q_{41} & 0 & q_{44} & 0 & 0 \\ 0 & 0 & 0 & 0 & q_{44} & 2 q_{41} \\ 0 & 0 & 0 & 0 & q_{14} & q_{11}-q_{12}\end{array}$

The piezo-optic tensor is a 4 th rank tensor, as the tensor of elastic constant $C_{i j}$ (in this case $C_{14}=C_{41}$ ).

With Dauphiné twinning $q_{14}$ and $q_{41}$ change sign. So for the electro-optic as for the piezo-optic effect, there are coefficients which change their sign with Dauphiné twinning, so it must be possible to use these effects to observe this twinning.

2. Application of an electric field. - When we apply an electric field $E_{x}$ along $O X$, the crystal becomes slightly biaxial, $O X$ remains an axis of the ellipsoid of refractive indices, the two other axes are rotated in the plane $Y O Z$ by an angle $\theta \sim n^{3} \cdot d_{14} E_{x} /(2 \Delta n)$ from $O X$ and $O Y$ (where $\Delta n$ is the birefringence). The principal indices are :

$$
\begin{aligned}
& n_{x}^{\prime}=n_{x}+\frac{n_{0}^{3}}{2} r_{111} E_{x} \\
& n_{y}^{\prime}=n_{y}-\frac{n_{0}^{3}}{2} r_{111} E_{x} \\
& n_{z}^{\prime}=n_{z} .
\end{aligned}
$$

For a free crystal and for a wavelenght of $5890 \AA$ [12], one has: 


$$
\begin{aligned}
& r_{111}=1.3 \times 10^{-8} \mathrm{esu}=0.43 \times 10^{-11} \mathrm{~cm} / \mathrm{V} \\
& r_{123}=0.7 \times 10^{-8} \mathrm{esu}=0.23 \times 10^{-11} \mathrm{~cm} / \mathrm{V} .
\end{aligned}
$$

There are two experimental possibilities. One can either observe the opposite rotation of the position of extinction from one twin to the other (the angle of rotation $\theta$ is quite small $(0.45 \mathrm{mrad}$ for $\left.\left.E_{x}=100 \mathrm{kV} / \mathrm{cm}\right)\right)$; or one can use the change of refractive indices to make observations by phase contrast or interferometry, or more simply by using birefringence. The light propagating with its wave vector $\mathbf{k}$ in the $Y O Z$ plane, has an ordinary polarization when the electric field of the light beam is along $O X$. The corresponding refractive index $n_{0}^{\prime}$ is :

$$
n_{0}^{\prime}=n_{x}+\frac{n_{x}^{3}}{2} d_{111} E_{x} .
$$

The ray with its polarization in the $Y O Z$ plane is extraordinary, with an index $n_{\mathrm{e}}^{\prime}$ given by :

$n_{\mathrm{e}}^{\prime}=n_{\mathrm{e}}(\varphi)+\frac{n_{\mathrm{e}}^{3}}{2}\left(-d_{111} \cos ^{2} \varphi+d_{123} \sin 2 \varphi\right) E_{x}$

where $\varphi$ is the angle between $\mathbf{k}$ and $O Z$. The change of refractive index $n_{\mathrm{e}}^{\prime}$ for a field $E=1 \mathrm{kV} / \mathrm{cm}$ as a function of angle $\varphi$ is plotted in figure 1. The change of birefringence is maximum for light propagating near $O Z$ (due to the disturbing effect of rotatory power it is better to have light propagating at some angle from $O Z$ ).

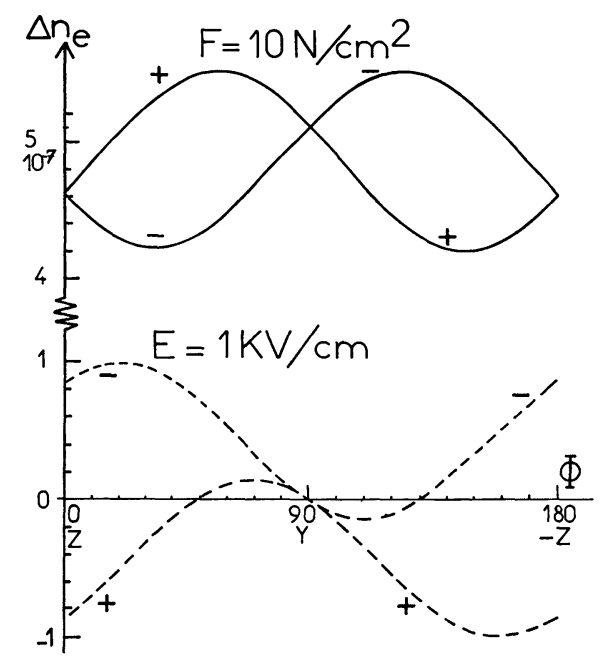

Fig. 1. - Angular dependence of the extraordinary refractive index change $\Delta n_{\mathrm{e}}$ of quartz, induced by piezooptic or electrooptic effects, for a light beam propagating in $Y O Z$ at an angle $\varphi$ from $O Z$.

The electric field can also be applied along $O Y$. The crystal becomes biaxial but the principal directions are now near $110,1 \overline{1} 0,001$. The magnitude of indices along $O X, O Y, O Z$ is not changed in first order. The induced birefringence is maximum for light propagating along $O Z$ with polarization along 110 and $1 \overline{1} 0$.
3. Application of an uniaxial stress. - The same kind of effects are produced by applying an uniaxial stress $\sigma$. If a compressive stress is applied along $O X$ the crystal becomes biaxial, with a small rotation $\theta$ of the principal direction in $Y O Z$ plane $(\theta=6.9 \mathrm{mrad}$ for a load of $10^{4} \mathrm{~N} / \mathrm{cm}^{2}$, but experimental observation is difficult because the light beam must be parallel to the direction of application of the force). The refractive indices change unequally in different twins. For light propagating in $Y O Z$ plane the refractive indices are :

$n_{0}^{\prime}=n_{x}+\frac{n_{x}^{3}}{2} q_{11} \sigma_{1}$

$n_{\mathrm{e}}^{\prime}=n_{\mathrm{e}}+\frac{n_{\mathrm{e}}^{3}}{2} \times$

$$
\times\left(q_{31}+\left(q_{12}-q_{31}\right) \cos ^{2} \varphi+q_{41} \sin 2 \varphi\right) \sigma_{1} .
$$

These changes of the extraordinary refractive indices are also plotted in figure 1 for a stress of $10 \mathrm{~N} / \mathrm{cm}^{2}$ using Pockel's value for $q_{i j}$ [2] $\quad\left(q_{11}=1.11\right.$; $q_{12}=2.50 ; q_{13}=1.97 ; q_{14} \pm 0.097 ; q_{31}=2.77$; $q_{33}=0.183 ; q_{44}=-1.015 ; q_{41}= \pm 0.32$; all $\times$ $10^{-8} \mathrm{~cm}^{2} / \mathrm{N}$.

A stress along $O Y$ also makes a twin-dependent small rotation of the principal axes in the plane $Y O Z$ which can be observed by sending light along $O X$. A stress along $O Z$ produces no twin-dependent effect.

One can also stress the sample with a force which is not parallel to crystallographic axes introducing shear stresses. For example a force directed along 011

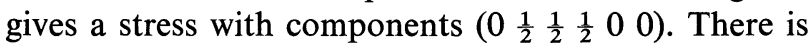
also a twin-dependent rotation of the principal axes in $Y O Z$ (this was the experimental configuration used by Aizu and Irai [7]) and the refractive indices $n_{x}$ and $n_{y}$ are changed by a twin-dependent quantity. Stress in $X O Y$ or $Y O Z$ plane can also be used but effects are more complicated, as the resultant ellipsoid is not invariant under the symmetry operation of the crystal.

4. Experimental results. - In the experimental work we have applied stress and electric fields only along $O X$. The experimental arrangement is shown in figure 2. A collimated light beam is obtained from a $15 \mathrm{~W}$ incandescent lamp. The sample is a $10 \times 10 \times 2 \mathrm{~mm}^{2}$ slab with two plane parallel polished faces. (BC cut, the normal is at $31^{\circ}$ from $O Z$.) One of the sides is perpendicular to $O X$ which is orientated vertically. A mechanical stress is applied along $O X$ by a lever with a load. The faces perpendicular to $O X$ were recovered with silver paint for the application of an electric field. As we work with white light it is necessary to use a compensator in order to equalize the optical path for the two perpendicularly polarized light components, so that one can observe extinction for all wave lengths simultaneously 


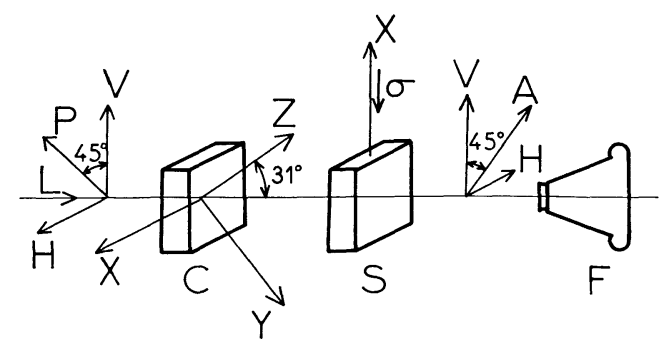

FIG. 2. - Experimental set-up for observing Dauphiné twins in a quartz sample under mechanical stress or electric field. $\mathbf{P}$ and $\mathrm{A}$ crossed polarizers at $45^{\circ}$ from vertical. $\mathrm{S}$ quartz sample with $X$ axis vertical, $C$ identical crystal with $X$ axis horizontal, used as a compensator. F photographic camera. A force $\sigma$ (or an electric field can be applied along $X$ axis on the sample), a white light beam $L$ is used to make observations.

between crossed polarisers (polaroid sheets). The compensator is made of a quartz slab identical to the sample but with its $O X$ axis horizontal. The optical paths for horizontally and vertically polarized light are the same when the two crystals are perpendicular to the light beam. The compensator can be rotated around $O X$, changing the retardation of the vertically polarized light and interference colors then appear on both sides of exact compensation. The crystal can be observed with a small microscope and photographed with a camera. When a force is applied along $O X$, the sample birefringence is changed and interference colors appear. By rotating the compensator a little the light can be extinguished. But with a stress greater than $500 \mathrm{~N} / \mathrm{cm}^{2}$ different Dauphiné twins cannot be extinguished exactly at the same time. For a stress of about $2500 \mathrm{~N} / \mathrm{cm}^{2}$ twins appear quite distinctly either near extinction (black and grey) or with interference color, the effect being more apparent for the sensitive tints (purple to red). The phase change between the two twins is about $30^{\circ}$. In general, twin walls have straight directions and fine structures can often be seen in the walls (Fig. 3).

The same observations can be made by applying an electric field along $O X$. As the electro-optic effect is quite small it is necessary to use high field $(40 \mathrm{kV}$ on $1 \mathrm{~cm}$ crystal).

The sample was placed in an oil bath to prevent electrical breakdown on the surfaces. Twins were also observed visually using the same samples as before,

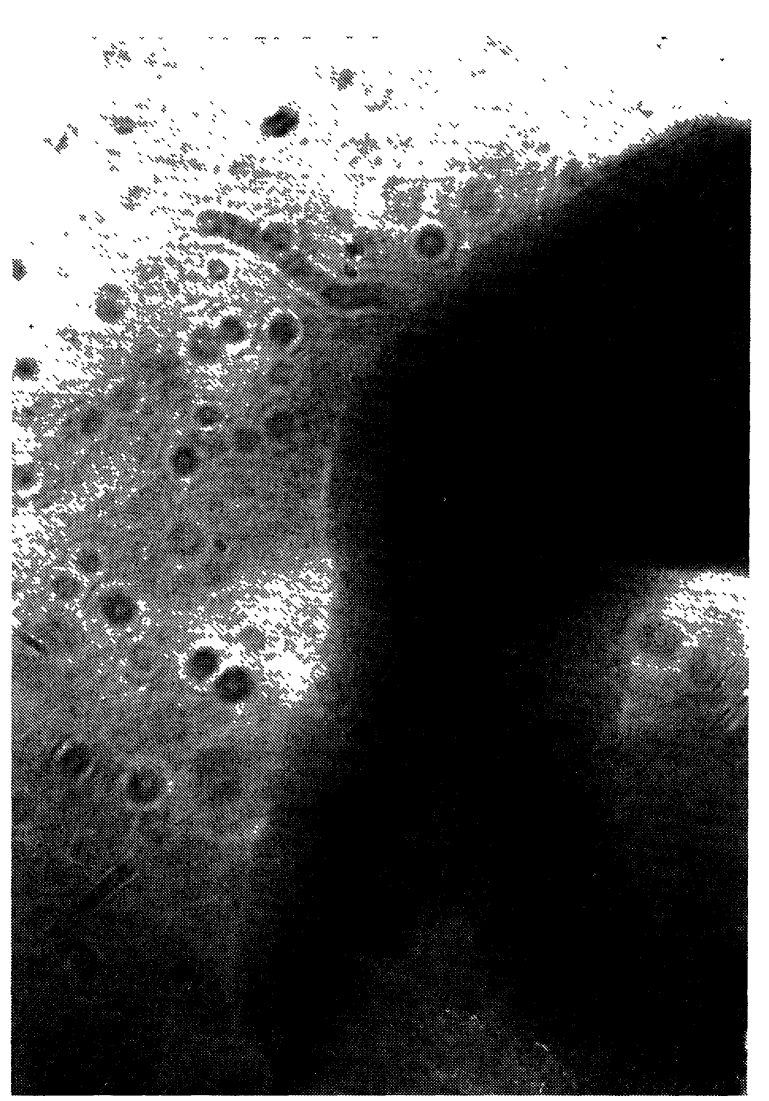

Fig. 3. - Photograph of Dauphiné twins in a quartz sample with an applied stress of $2500 \mathrm{~N} / \mathrm{cm}^{2}$ along $O X$, vertical direction. The area of crystal photographed here is about $3 \times 4.5 \mathrm{~mm}^{2}$. The $O X$ axis of the light area has opposite orientation relative to the $O X$ axis of the dark area (Dauphiné twinning).

but with bad contrast, so that we were not able to take good pictures.

We have shown that it is possible to use electrooptic and piezo-optic effects, the last with good results, in order to observe Dauphiné twins in quartz. As a result of the natural birefringence it is necessary to have crystals with parallel faces optically polished. This is a limitation to the use of this method for systematic control.

Acknowledgements. - I am pleased to acknowledge the technical assistance of P. Palleau for the experimental part of this work.

\section{References}

[1] Frondel, C., Dana's system of mineralogy (Wiley, New York) 1962, 7th ed. Vol. III.

[2] Cady, W. G., Piezoelectricity (Dover Publications, New York) 1964.

[3] Pascal, P., Nouveau traité de Chimie Minérale. VIII-Silicium (Masson et Cie, Paris) 1965.

[4] Lang, A. R., Appl. Phys. Lett. 7 (1965) 168.

[5] Maclaren, A. C. and Phakey, P. P., Phys. Stat. Sol. 31 (1969) 723.

[6] Dolino, G., Bachheimer, J. P. and Vallade, M., Appl. Phys. Lett. 22 (1973) 623.
[7] Aizu, K., J. Phys. Soc. Japan 34 (1973) 121.

[8] Friedel, G., Leçons de cristallographie (Librairie Scientifique Albert Blanchard, Paris) 1964.

[9] Bloembergen, N., Nonlinear Optics (Benjamin, New York) 1966.

[10] Aizu, K., J. Phys. Soc. Japan 32 (1972) 1287.

[11] Bachheimer, J. P. and Dolino, G., Phys. Rev. B 11 (1975) 3195.

[12] Veerabhadra RaO, K., and Narasimhamurty, T. S., $O p t$. Acta 19 (1972) 319. 